\title{
A STOKESIAN DYNAMICS APPROACH FOR SIMULATION OF MAGNETIC PARTICLE SUSPENSIONS
}

\author{
Anders Sand ${ }^{* 1}$, Jan F. Stener ${ }^{1}$, Martti O. Toivakka ${ }^{3}$, Johan E. Carlson ${ }^{2}$ and Bertil I. Pålsson ${ }^{1}$ \\ ${ }^{1}$ Minerals and Metallurgical Research Laboratory \\ Dept. of Civil, Environmental and Natural Resources Engineering \\ Luleå University of Technology \\ SE-971 87 Luleå, SWEDEN \\ ${ }^{2}$ Division of Signals and Systems \\ Dept. of Computer Science, Electrical and Space Engineering \\ Luleå University of Technology \\ SE-971 87 Luleå, SWEDEN \\ ${ }^{3}$ Laboratory of Paper Coating and Converting, Centre for Functional Materials \\ Faculty of Science and Engineering \\ Åbo Akademi University \\ FI-20100 Turku, FINLAND \\ *Anders.Sand@1tu.se
}

Keywords: Particle dynamics, magnetic interactions, mineral suspensions, simulation.

\begin{abstract}
The dynamic behaviour of $\mu \mathrm{m}$-scale ferromagnetic particles in suspension is relevant for various mineral beneficiation processes. It is, however, difficult to experimentally study such processes at the particle-level. In these instances it can be advantageous to resort to suitable particle simulation methods.
\end{abstract}

Stokesian dynamics is a mesh-free numerical technique developed for suspensions of $\mathrm{nm}$ to $\mathrm{mm}$ size particles. The method inherently considers hydrodynamic interactions, but additional interaction models can be included depending on the system under investigation. We here 
present a Stokesian dynamics (SD) implementation, which allows for simulation of the motion of suspended magnetic particles in presence of an external magnetic field. The magnetic interaction model includes particle-field interactions as well as pairwise interactions between magnetised particles.

Simulations are compared with experiments using a laboratory-scale flow cell. The method is shown useful for studying ferromagnetic suspensions in mineral processing applications, and for understanding and predicting the efficiency of mineral separation processes.

\section{INTRODUCTION}

The study of magnetic particle suspensions is relevant for better understanding of wet-state separation processes in mineral processing and recycling (Stener et al., 2014; Menad et al., 2014; Svoboda and Fujita, 2003) as well as various other applications or processes based on magnetic properties of particles, for instance magnetorheology, micro-robotics, and medical applications (Costa and Costa Banco, 2009; Lu et al., 2014; Rosengart et al., 2005; Lunov et al., 2011).

Numerical simulation of magnetic particles in suspension have been conducted within a number of probabilistic and deterministic particle dynamics simulation disciplines, including MonteCarlo simulation (Satoh et al., 1996; Satoh, 2008), LB-DEM (Han et al., 2010a; 2010b), and Stokesian dynamics (Satoh et al., 1998; Satoh, 2002; Irisa and Yokomine, 2007). The focus of these studies have not been directed towards mineral separation processes, but rather been phenomenologically oriented or related to novel uses of magnetic effects in applications related to for instance nanotechnology, ferrofluids, and drug delivery systems. One of the more accurate methods related to evaluating the combined effect of liquid phase flow and magnetic effects is the LB-LES-DEM approach (Lattice-Boltzmann-Large Eddy Simulation-Discrete Element Method). Work in this direction has been described by Han et al. (2007; 2010a; 2010b). The 
method is, however, restricted to a very limited number of particles due to the high computational expense.

Some relatively recent studies have been carried out utilising combined DEM-FEM-CFD simulation approaches for simulating high-gradient magnetic separation (HGMS) and wet lowintensity magnetic separation (LIMS). Such work has been published by Lindner et al. (2013) and Murairu (2013). These types of coupled methods are beneficial in offering one or sometimes two-way coupling between continuum and discrete simulation disciplines by using commercially available simulation tools. The drawbacks mainly come from inherent limitations of each individual method or the coupling between the methods. This can be exemplified by the typical one-way hydrodynamics coupling or otherwise simplified description of the interparticle hydrodynamics, limited possibilities to describe free surface interactions, etc. Although enabling the simulation of fairly large systems and allowing a relatively accurate representation of the macroscopic magnetic field, these methods are not able to fully account for the complex mechanisms that can arise as result of combined effects of magnetic, hydrodynamic and possibly other relevant particle-level interactions.

In processes such as magnetic separation, single particle properties and interactions play a key role. It is therefore strongly motivated to build such work on predominantly particle-oriented simulation approaches, where particles can be individually designated relevant properties and various mechanisms can be reliably studied at the particle level. A benefit of the Stokesian dynamics technique is that it is developed for particles in suspensions and thus allows for study of high-concentration suspension behaviour without need for the computationally more costly DEM-CFD or DEM-FEM coupling or less accurate tracer particle simulations by using continuum-type approaches. The Stokesian dynamics technique allows relatively straightforward addition of force models describing various types of particle interactions in liquid media (Toivakka et al., 1997; Nopola, 2004). 
In this paper we propose a Stokesian dynamics-based simulation framework, which can combine the accuracy of suspension-oriented particle methods with the efficiency of a simplified mesh-free mathematical approximation of the magnetic field and fluid flow. The model is illustrated and qualitatively evaluated in the context of a number of simulation examples. Furthermore, simulations are compared with experimental results using a laboratoryscale flow cell. We also discuss the wider applicability to the model for numerical simulationbased evaluation of magnetic separation efficiency.

\section{METHOD}

\subsection{Stokesian Dynamics}

The Stokesian dynamics technique was originally developed in the 1980's for the study of many-body interactions in non-equilibrium suspensions (Bossis and Brady, 1984; Brady and Bossis, 1988; Brady, 2001). At that time, the main focus of simulations was directed towards studying the behaviour of hydrodynamically interacting particles in suspensions under various flow conditions. Due to the possibility to include additional interaction models, Stokesian dynamics implementations have since then been developed to, for instance, enable consideration of colloidal interactions, Brownian motion, and interparticle effects from added dispersant (Toivakka et al., 1997; Nopola et al., 2004; Sand et al., 2009). There has also been much work devoted to increasing the computational efficiency of the technique (e.g. Bousfield, 1990; Sierou and Brady, 2001).

Earlier approaches using the Stokesian dynamics technique for studying ferromagnetic particle suspensions have included the definition of the external magnetic field as a homogeneous field covering the full simulation domain, as well as describing particles as being identical and of monodisperse size distribution (Satoh et al., 1998; Irisa and Yokomine, 2007). Efforts have also been made to reduce the computational cost by introduction of a cluster-based simulation approach (Satoh, 2002). 
Stokesian dynamics simulations are based on the N-body coupled Langevin equation (Brady and Bossis, 1988), which is a variant of Newton's second law of motion. It can be written in the form

$$
\mathbf{m} \frac{d \mathbf{U}(\mathbf{t})}{d t}=\mathbf{F}^{\mathbf{H}}+\mathbf{F}^{\mathbf{P}}+\mathbf{F}^{\mathbf{M}},
$$

where mass, $\mathbf{m}$, times the change in velocity, $\frac{d \mathbf{U}(\mathbf{t})}{d t}$, is a function of the various forces influencing the particles. These forces are commonly divided into hydrodynamic forces, $\mathbf{F}^{\mathbf{H}}$, interparticle forces, $\mathbf{F}^{\mathbf{P}}$, and single particle forces such as for instance resulting from macroscopic forces of varying origins which influence particles, $\mathbf{F}^{\mathbf{M}}$. Due to the additivity of forces in this expression, interactions beyond the typical hydrodynamics and particle interaction models can be included in a relatively straightforward manner. For reducing the computational expense, it is often necessary to conduct an analysis of governing forces through the calculation of non-dimensional numbers. This analysis includes a relative comparison between different forces in order to determine their relative significance. In Stokesian dynamics, the most important non-dimensional number is the particle Reynolds number, $R e_{p}$. It describes the relative importance of inertial versus viscous effects as

$$
R e_{p}=\frac{\rho_{l} a_{c h a r} u_{c h a r}}{\mu}
$$

where $\rho_{l}$ and $\mu$ are the density and viscosity of the liquid medium, respectively, $a_{c h a r}$ the characteristic particle size and $u_{\text {char }}$ the velocity of particles relative to the liquid. If the particle Reynolds number is small, defined as $R e_{p} \ll 1$, it can be assumed that viscous effects dominate and particles can be expected to react instantaneously to forces influencing them. This approximation is relevant for small particles that are freely moving with the fluid, and thus suitable for suspension flow simulations. For instance a $1 \mu \mathrm{m}$ particle moving at a velocity of $0.1 \mathrm{~m} / \mathrm{s}$ relative to the liquid phase (water) has a particle Reynolds number of 0.1 . 
While this form of numerical analysis is relatively straightforward in steady-state-type systems, it becomes complicated in systems with rapidly changing conditions or where conditions vary considerably at different locations of the domain. This can for instance be assumed to be the case near the magnetic source in the simulations presented in this work, as in this region magnetic interaction forces will dominate.

In addition, the Stokesian dynamics approach utilised in this work includes CFD-adaptations to the hydrodynamics model expressions in order to improve the accuracy of simulations when studying particle systems of wider particle size distributions than the $1 / 10$ size ratio traditionally considered as a limitation in Stokesian dynamics (Kim and Karrila, 1991). This adaptation is described in detail by Sand et al. (2009) and Nopola (2004).

\subsection{Magnetic Interactions}

In this work, we aimed at creating a flexible implementation for simulation of ferromagnetic particle suspensions under influence of three-dimensional magnetic fields with an arbitrarily assignable magnetic source. This is advancing the previous state-of-the-art in Stokesian dynamics simulations, where suspension behaviours have been studied in simplified, homogeneous magnetic fields (Satoh et al., 1996; Satoh, 2002; Irisa and Yokomine, 2007).

Particle motion and interactions related to magnetic effects are in this work based on three force models. This includes interaction between the particles and a stationary magnetic point source, an interparticle magnetisation model and a magnetic particle cluster orientation model. There are a number of assumptions made in order to increase the computational efficiency:

- Particle magnetisation is assumed to be homogeneous, and vary depending on the local magnetic field, which is a function of the distance to the magnetic point source. The magnetic point source is furthermore viewed as an infinitely long dipole. 
- Particles are assumed to always be oriented in the direction of the magnetic field, as defined by the position of the magnetic point source. Consequently, particles are not specifically described as magnetic dipoles. Their magnetic dipole orientation is therefore always known and calculation of magnetic torque on individual particles can be omitted. Since particles in suspension have a small rotational drag this is deemed a reasonable approximation.

- Particles which are defined as being in close contact with each other in the vicinity of the magnetic pole are considered as being part of a magnetic cluster, whereby an additional force model works to orientate the particle cluster with the magnetic field. This can be considered as a cluster-based torque model, which applies for particle pairs but not for individual particles.

- No back-coupling is assumed between magnetised particles and the macroscopic magnetic field, i.e. magnetised particles do not explicitly affect this field. The localised effect that these particles have on the field is instead considered through the interparticle magnetic interaction and cluster orientation models.

The relationship between magnetic susceptibility, $\mathbf{S}$, the intensity of magnetisation, $\mathbf{M}$, and the applied magnetic field, H, for various materials is discussed by Wills and Napier-Munn (2006). While the volume magnetic susceptibility of paramagnetic and diamagnetic materials is nearly a constant, the relation between $\mathbf{M}$ and $\mathbf{H}$ for ferromagnetic materials is more complex. Modelling of magnetisation curves has previously been attempted (Abuelmaatti, 1993), but due to the relative complexity of these expressions a simplified description was estimated for use in this work. In order to produce a realistic magnetisation behaviour of ferromagnetic particles, it was therefore assumed that the intensity of magnetisation of a single particle, $\mathbf{M}$, is a function of the saturation magnetisation, $\mathbf{M}_{\mathbf{S}}$, a magnetisation gradient, $g$, and ambient magnetic field $\mathbf{H}$ according to

$$
\mathbf{M}=\mathbf{M}_{\mathbf{S}}\left|e^{-g \mathbf{H}}-1\right|
$$


Particle magnetisation based on their distance from the magnetic point source is assumed to follow this expression. As can be seen, this does not include any memory function for the magnetisation of particles. A comparison between the magnetisation model and experimental data is made in Figure 1. The magnetisation intensity and applied magnetic field $(\mathrm{T})$ can be expressed in SI-units $(\mathrm{A} / \mathrm{m})$ by dividing with $4 \pi \times 10^{-7} \mathrm{~T} / \mathrm{Am}^{-1}$.

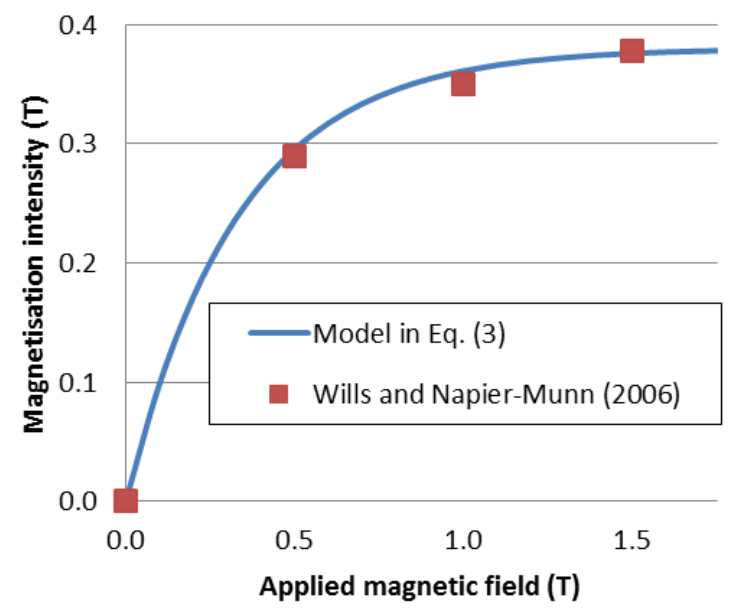

Figure 1. Magnetisation curve of ferromagnetic material (magnetite) with saturation magnetisation $0.38 \mathrm{~T}$ based on Eq. (3), as compared with data provided by Wills and NapierMunn (2006).

The magnetic dipole-dipole interaction force between two nearby particles can be defined as

$$
\mathbf{F}_{a b}=\frac{3 \mu_{0}}{4 \pi|r|^{4}}\left[\left(\mathbf{r} \times \mathbf{m}_{a}\right) \times \mathbf{m}_{b}+\left(\mathbf{r} \times \mathbf{m}_{b}\right) \times \mathbf{m}_{a}-2 \mathbf{r}\left(\mathbf{m}_{a} \cdot \mathbf{m}_{b}\right)+5 \mathbf{r}\left(\left(\mathbf{r} \times \mathbf{m}_{a}\right) \cdot\left(\mathbf{r} \times \mathbf{m}_{b}\right)\right)\right]
$$

Where $|\mathbf{r}|$ is the distance between the two magnetic dipole moments, $\mathbf{m}_{a}$ and $\mathbf{m}_{b}, \mathbf{r}$ the unit vector pointing from $\mathbf{m}_{a}$ to $\mathbf{m}_{b}$ (Yung et al., 1998). The implementation of the model is simplified by assuming that the particles are always oriented in direction of the external magnetic field. The relative (non-dimensional) magnitude and direction of the force between 
two magnetically interacting particles in a homogeneous magnetic field is for illustration shown in Figure 2.
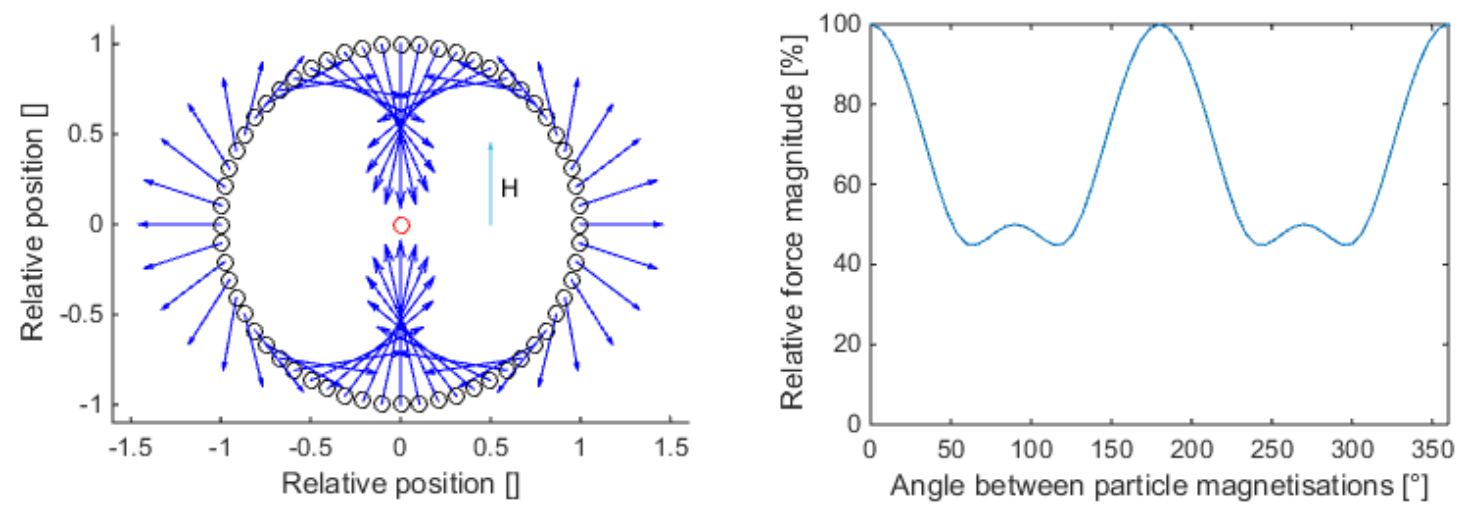

Figure 2. Direction (left) and relative force magnitude (right) of interaction between two magnetised particles in an ambient magnetic field $(\mathbf{H})$.

\subsection{Algorithmic techniques}

Various algorithmic techniques are typically incorporated in Stokesian dynamics codes in order to improve the computational efficiency of simulations. The implementations used in this work include a sparse matrix solver as well as a neighbour list technique which allows the calculation of only pairwise interaction of nearby particles. This significantly reduces the computational efforts (Toivakka, 1997; Sand et al., 2009). However, long-range interactions originating from the macroscopic magnetic field is not compatible with the neighbour list approach. In the algorithmic implementation, the attraction of particles to the magnetic source is instead considered as a single particle force. Interparticle forces resulting from magnetisation and particle cluster orientations are still taken into consideration within the neighbour list approach. This admits the calculation of long-range magnetic attraction forces simultaneously as the numerical implementation for particle-level interactions remains relatively efficient. 


\subsection{Laboratory-Scale Flow Cell}

The setup of simulation examples presented in this work was constructed to allow comparison and later possibly validation with a laboratory scale flow cell, which can be equipped with an ultrasound transducer and magnets. The dimensions of the flow cell are 50x75 mm (depth and width, respectively) and the length is $1000 \mathrm{~mm} .50 \mathrm{~mm}$ is a typical distance between the magnetic drum and the tank bottom in wet LIMS, while $75 \mathrm{~mm}$ is wide enough to avoid unwanted interaction between the ultrasound signal and the side walls. The ultrasound transducer enables the tracking of flow profile and localised solids concentrations in the suspension. If equipped with magnets, magnetic material can be captured at the wall of the flow cell. This allows the monitoring of magnetic material build-up near the magnet. During build-up it is possible to study, for instance, rate of build-up, or cake thickness as function of flow rate. Experimentally tested suspensions within this flow cell have included magnetite particles, $\mathrm{d}_{50}=34 \mu \mathrm{m}$, and at a concentration of $5-10 \mathrm{vol} \%$ at a suspension flow rate of up to $1 \mathrm{~m} / \mathrm{s}$ (Stener et al., 2014). The flow cell setup is schematically shown in Figure 3.

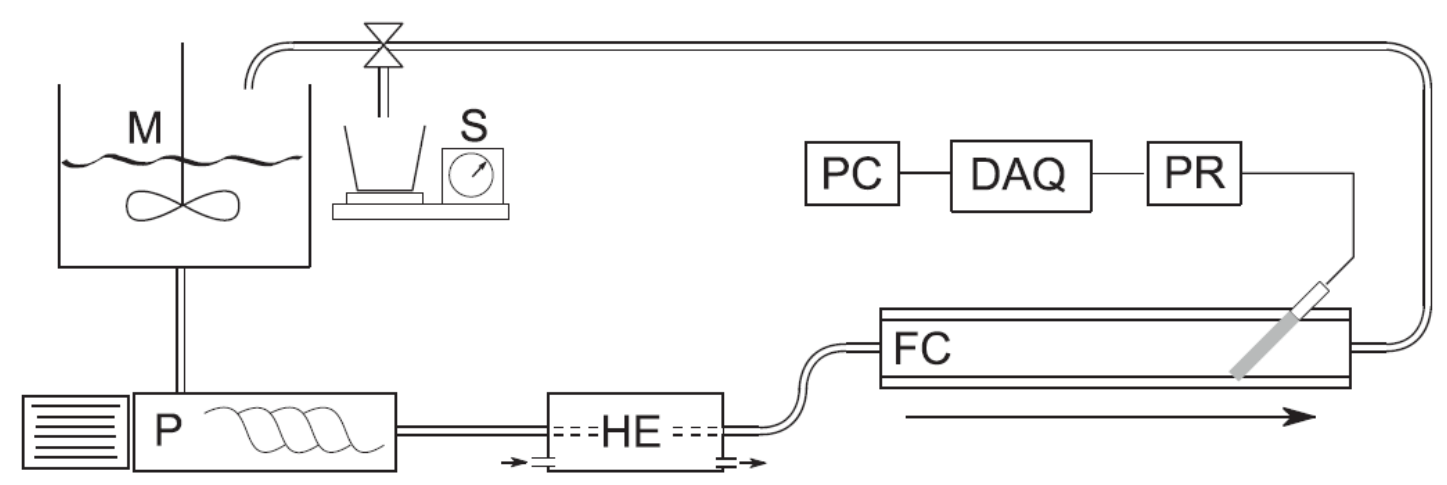

Figure 3. Experimental flow cell setup, including mixer (M), pump (P), heat exchanger (HE) and flow cell (FC). The flow cell can be equipped with an ultrasound transducer as well as magnets at the same position (Stener et al., 2014). 


\section{RESULTS}

\subsection{Simulation Setup}

The simulation examples given below are designed to roughly correspond to the setup of the described flow cell. A pressure-driven (parabolic) flow profile is used to describe the flow of the suspension. Solids concentrations up to 13 vol\% have been successfully simulated, which is comparable to the maximum slurry concentrations used in the flow cell and also similar to the conditions in a magnetic separator. Due to the large number of particles (billions per $\mathrm{cm}^{3}$ ) in the flow cell, a full representation of the particle system within a simulation domain is not possible with current computational resources. Instead, the simulations below should be considered as representing only a small volume of the cell, typically less than $0.1 \mathrm{~mm}^{3}$. Only monodisperse systems of spherical particles are considered in simulations, with particle sizes of $2-5 \mu \mathrm{m}$. The Stokesian dynamics approach presented in this work can, however, also be utilised for simulation of particle size distributions.

\subsection{Simulation Examples}

The above-described simulation approach is evaluated in a number of test cases to demonstrate the functionality of the constituent models and how they interact. Results are visualised using the in-house developed software PiMP++, based on the OpenGL platform (Sand, 2010).

Tracking of particle motion can aid in the understanding of capture criteria and near-magnet flow patterns based on the combined effect of hydrodynamics, magnetic, and other relevant forces. An example of such a simulation is shown in Figure 4. Particles have at the start of the simulation been randomly placed in the simulation domain and a parabolic flow profile applied. It can be seen that particles close to the defined magnetic point source are able to migrate against the flow direction towards the magnet. If the distance to the magnet source is too long, particles attracted to it might not be able to reach it before being carried away with the flow. 
This is especially noted if particles need to pass the high flow velocity region in the centre of the flow channel.

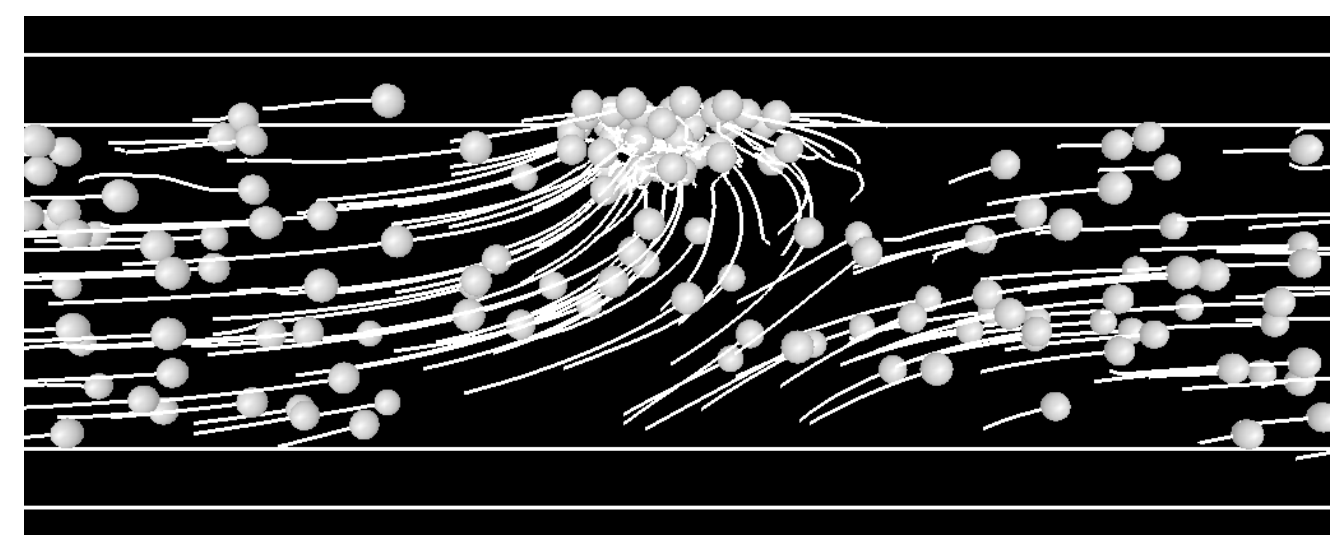

Figure 4. Magnetic particles and their trajectories near a magnetic pole. The straight white lines mark the inner corners of the flow cell.

It is known that non-magnetic particles in some cases can be captured within magnetic particle clusters and consequently carried with the concentrate (Garcia-Martinez et al., 2011). The mechanisms of such non-magnetic particle entrapment is not known in detail, but can be studied by numerical simulation, for instance by investigating the interrelation between macroscopic suspension flow, interparticle hydrodynamic effects, and magnetic forces. Stokesian dynamics is a highly suitable technique in this respect as it includes full representation of hydrodynamic particle interactions through the liquid medium, while typical approaches based on CFD-DEM or FEM-DEM assume that particles move along the liquid flow lines (Murariu 2013), but do not interact hydrodynamically with each other. This is especially relevant in high-concentration suspensions, where the calculation of hydrodynamic interactions in Stokesian dynamics strongly contributes to increased simulation accuracy (Nopola, 2004). It also needs to be noted, however, that neither this approach nor the CFD-DEM or FEM-DEM-work discussed above include twoway coupling between the liquid phase and particles. 
Another simulation was carried out with a suspension of 1000 magnetic and an equal number of non-magnetic particles, Figure 5. Two distinct mechanisms that could result in particle entrapment were observed. The first being non-magnetic particles getting dragged or pushed by hydrodynamic or mechanical interactions with magnetic particles translating towards the magnetic source. The second mechanism was the flow of non-magnetic particles towards the formed magnetic cake by simple hydrodynamic drag by the flowing liquid medium. When suspended magnetic particles approach from behind and lock into the magnetic particle cluster, they may completely surround the non-magnetic particle before it has the chance to escape.

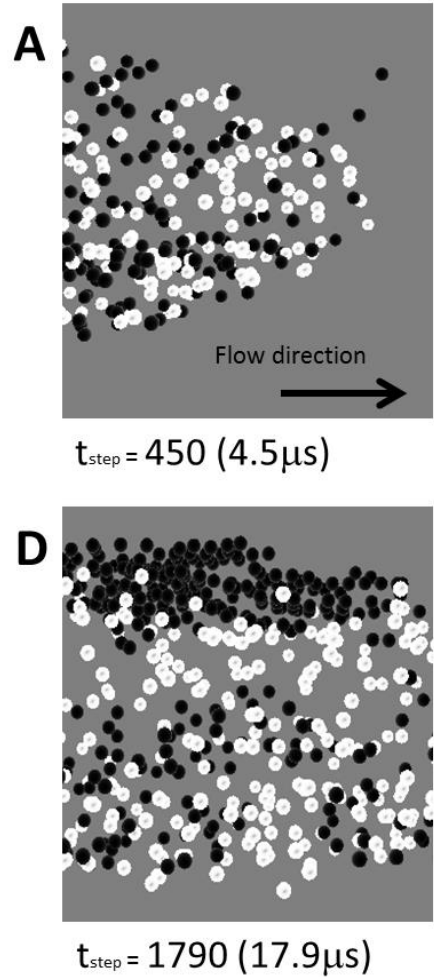

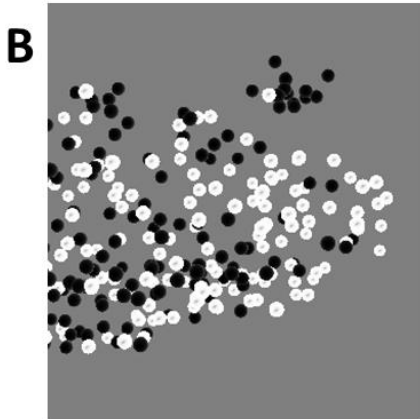

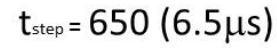

E

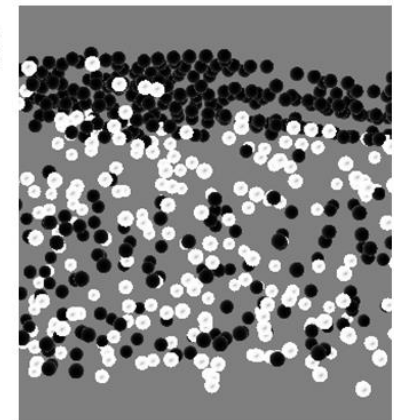

$\mathrm{t}_{\text {step }}=2030(20.3 \mu \mathrm{s})$
C

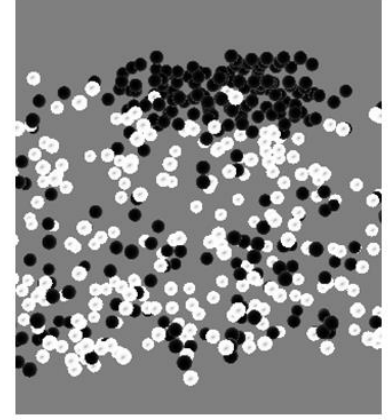

$t_{\text {step }}=1360(13.6 \mu \mathrm{s})$

$\mathbf{F}$

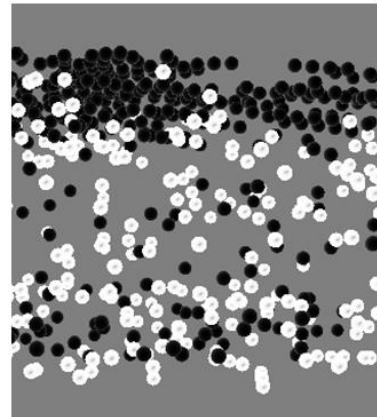

$\mathrm{t}_{\mathrm{step}}=2400(24.0 \mu \mathrm{s})$

Figure 5. Motion of $2 \mu \mathrm{m}$ magnetic particles (dark) and non-magnetic particles (light). The later time steps show entrapment in the particle clusters near the magnetic pole.

In addition to particle entrapment, the simulation also shows the formation of chains of magnetic particles, which orientate in the direction of the flow. This is a result of particle magnetisation and consequently attraction of particles to each other in the vicinity of the 
magnetic pole. Strings of magnetic particles appear under some conditions to be able to eventually detach from the structure as seen in subfigures E and F.

As the number of particles in simulations is relatively low, an alternative type of visualisation was tested. Instead of visualising individual particles, sticks were used to illustrate particles in close contact to each other. The position of sticks can then in turn be viewed as a high concentration region of particles or as an indicator of particle aggregation due to magnetic or other forces. Such an example is shown in Figure 6. The structure arising in simulations can be compared with the visually observable magnetic cake in an experiment using the flow cell shown in Figure 3. Results appear very similar in terms of magnetic cake structure, with a strong build-up near the magnet and a tail of particles in the direction of the flow. It needs to be pointed out that since only particle clusters are illustrated in this example, individual particles that are not considered as forming part of a cluster are not at all shown using this mode of visualisation.
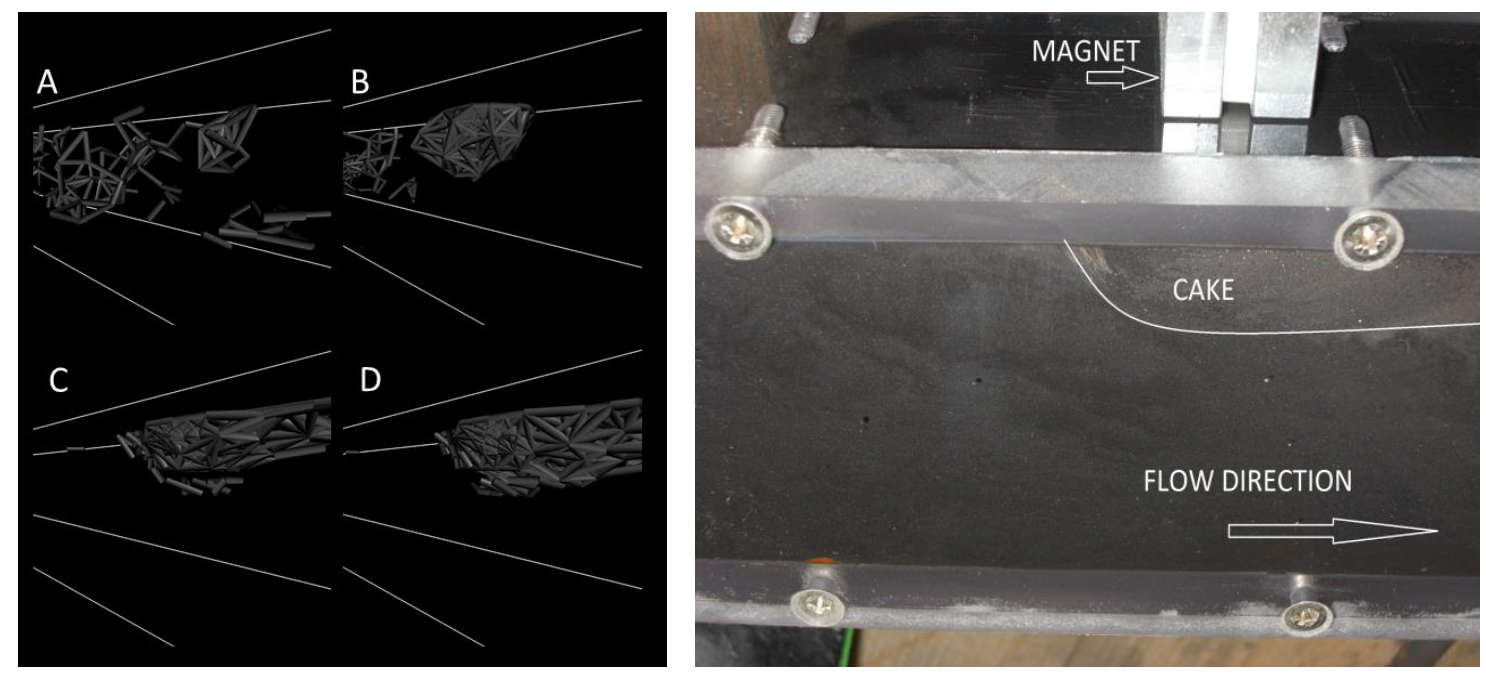

Figure 6. Visualisation of particle contacts as sticks (left). Magnetic material flowing through the cell captured by the magnet (A), cake build-up (B and C) and a steady state-type condition reached (D). Simulations can be compared with the equilibrium shape of the particle cake formed in the flow cell (right). 
By adjusting the threshold distance at which particle contacts are filtered out, it is possible to adapt the visualisation to illustrate individual clusters of magnetic particles in close-contact rather than the general increase in solids concentration shown in the previous example. An example of this is given in Figure 7, where contacts between individual particles are shown as sticks with increased thickness at the interparticle gap. This gap can in practice be determined by a specified particle surface roughness parameter. The particle chains are formed as a result of the interparticle magnetisation, the particle cluster orientation model which adapts to the field lines extending from the magnetic pole and various hydrodynamic interactions.

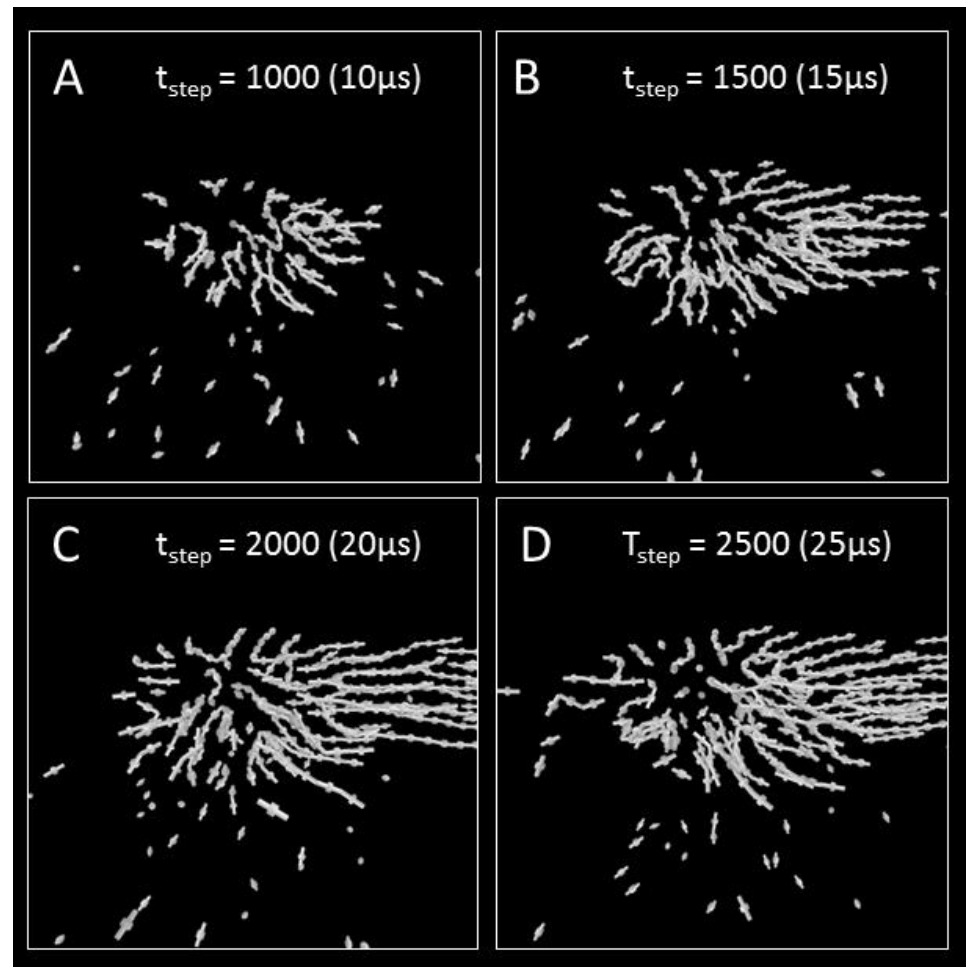

Figure 7. Time lapse showing formation of chainlike magnetic clusters at the magnetic pole, visualised as connections between particles in close contact.

The four examples discussed above illustrate numerical results along with a number of visualisation cases that can be produced using the method developed in this work. The simulation outcomes are logical in comparison with previous simulation work, experimental 
results and theory discussed in the introduction and method. Consequently, Stokesian dynamics appears to be a suitable method for simulation of magnetic phenomena in suspensions. With further development, the method is expected to allow relatively comprehensive studies to be carried out, e.g. related to mechanisms and interactions in magnetic separators. This could for instance include the effects of changes in material throughput, solids concentration and magnetic field strength on the selectivity of magnetic separation processes.

\section{CONCLUDING REMARKS}

In this work, we have adapted a model for magnetic interactions to a Stokesian Dynamics simulation framework. This gives the capability to study the three-dimensional behaviour of ferromagnetic particle suspensions of varying particle size distributions subjected to a magnetic field.

This work addresses some of the issues of previously conducted modelling and simulation work related to magnetic interactions in suspensions. These include lacking or having simplified descriptions of particle hydrodynamic interactions, limitation to monodisperse systems or the representation of the magnetic field. These simplifications bear the risk of seriously restricting the predictive value of earlier methods, especially with relation to particle interaction mechanisms resulting from the combination of magnetic and hydrodynamic forces. This strongly motivates the use of a Stokesian dynamics approach and the modifications suggested in this work, which allows more accurate hydrodynamic representation for polydisperse particle size distributions (Sand et al., 2011).

The model allows systematic study of various mechanisms or properties related to ferromagnetic suspensions, which in turn can have an influence on efficiency or selectivity of mineral separation processes. This can for instance include tracking of particle motion, study of 
the microstructure of the magnetic particle matrix and capturing mechanisms of non-magnetic particles. The examples presented in this work should, however, not be considered as a validation of the models, but rather a demonstration that reasonable results can be obtained. Due to the difference in particle system size and volume represented, simulations are not ideally corresponding to the flow cell setup and any study of differences or similarities between the two systems should therefore be restricted to qualitative comparisons. Given the considerations above, the examples demonstrated in this work is thus to be considered as giving indications of model capabilities rather than quantitative information on mechanisms or events taking place in the flow cell or in real magnetic separators.

A comparison between the approach discussed in this paper and work by other groups is made in Table 1.

Table 1. Comparison between the presented approach and some existing simulation techniques developed for magnetic interactions in suspensions

\begin{tabular}{|c|c|c|c|}
\hline & $\begin{array}{l}\text { Satoh et al. } \\
1999-2002\end{array}$ & $\begin{array}{c}\text { Murairu } \\
2013\end{array}$ & This method \\
\hline Simulation discipline & Stokesian Dynamics & $\begin{array}{l}\text { DEM-FEM-CFD } \\
\text { (uncoupled) }\end{array}$ & Stokesian Dynamics \\
\hline Dimensions & $3 \mathrm{D}$ & 3D-slice & $3 \mathrm{D}$ \\
\hline Hydrodynamics & Standard SD & Stokes drag & Improved SD \\
\hline $\begin{array}{l}\text { Particle-fluid back- } \\
\text { coupling }\end{array}$ & No & No & No \\
\hline Number of particles & 100 ’s & 100.000 's & 1000 ’s \\
\hline $\begin{array}{l}\text { Particle size } \\
\text { distribution }\end{array}$ & No & Yes & Yes \\
\hline
\end{tabular}




\begin{tabular}{|c|c|c|c|}
\hline Flow type & Laminar & Primitive turbulent & $\begin{array}{l}\text { Laminar or primitive } \\
\text { turbulent }\end{array}$ \\
\hline
\end{tabular}

\subsection{Further work}

Thus far, an approach with either completely magnetic or non-magnetic particles has been implemented and tested. The method is thus currently not adapted for studying mixed grain type particles. In processes where single particle properties play a role, for example liberation of magnetic material from gangue and magnetic grade effects in magnetic separation, it is crucial to be able to define individual particle properties in the models. Further work should thus include the development of interaction models where the size of the magnetic domain could be varied, for instance based on particle size. A long term possibility could be to also implement size distributions of the magnetic domain for different particle size classes or by assignment of magnetic properties to individual particles by random procedures.

So far, the work has been qualitatively compared with flow cell experiments (Stener et al. 2014). Further systematic comparison or validation type tests could include more detailed comparison of particle solids concentration or structure near the location of the external magnet. This would allow evaluation of the implemented model and possibly also adjustment of models or model parameters in order to produce a more accurate behaviour. Another possibility could be to quantitatively study non-magnetic particle capturing within the matrix of magnetic particles. This would however require a possibility to extract the magnetic cake from the flow cell and analyse the fraction of non-magnetic particles within. 


\section{ACKNOWLEDGEMENT}

Financial support to the project WET LIMS - Measurements and models, by the Hjalmar Lundbohm Research Centre (HLRC), is gratefully acknowledged. Luossavaara-Kiirunavaara Ab (LKAB) is acknowledged for supplying raw materials and access to pilot plant facilities.

\section{REFERENCES}

Abuelmaatti, M. (1993). Modeling of Magnetization Curves for Computer-Aided Design, IEEE Transactions on Magnetics 29(2):1235-1239.

Bossis, G. and Brady, J. (1984). Dynamic Simulation of Sheared Suspensions I. General Method, Journal of Chemical Physics 80(10), 5141.

Bousfield, D. (1990). The Simulation of Pigment Motion during Blade Coating, TAPPI Coating Conference, May 13-16, Boston, MA, 325.

Brady, J. (2001). Computer simulation of viscous suspensions, Chem. Eng. Sci. 56(9), 29212926.

Brady, J. and Bossis, G. (1988). Stokesian Dynamics, Annu. Rev. Fluid Mech. 20, 111-157.

Costa, E. and Costa Branco P. (2009). Continuum electromechanics of a magnetorheological damper including the friction force effects between the MR fluid and device walls: Analytical modelling and experimental validation, Sensors and Actuators A: Physical 155(1): 82-88.

Garcia-Martinez, H., Song, S., and Lopez-Valdivieso, A. (2011). In situ observation of quartz particles entrained into magnetic coagulates in a uniform magnetic field, Minerals Engineering 24(8), 963-966. 
Han, K., Feng, Y. and Owen, D. (2007). Coupled lattice Boltzmann and discrete element modelling of fluid-particle interaction problems, Computers and Structures 85: 1080-1088.

Han, K., Feng, Y. and Owen, D. (2010a). Three Dimensional Modelling and Simulation of Magnetorheological Fluids, Int. J. Numer. Meth. Engng. 84(11), 1273-1302.

Han, K., Feng, Y. and Owen, D. (2010b). Modelling of Magnetorheological Fluids with Combined Lattice Boltzmann and Discrete Element Approach, Commun. Comput. Phys. 7(5), 1095-1117.

Irisa, H., and Yokomine, T. (2007). Stokesian Dynamics Simulation of Nano-Magnets under External Magnetic Field, Int. Conf. ICIC, September 5-7, Kumamoto, Japan.

Kim, S. and Karrila, S. (1991). Microhydrodynamics - Principles and Selected Applications, Butterworth-Heinemann, Boston, MA, ISBN 0-7506-9173-5.

Lindner, J., Menzel, K. and Nirschl, H. (2013). Simulation of magnetic suspensions for HGMS using CFD, FEM and DEM modeling, Computers and Chemical Engineering 54, 111121.

Lu, Z., Poletkin, K., den Hartogh, B., Wallrabe, U. and Badilita, V. (2014). 3D micromachined inductive contactless suspension: Testing and modeling, Sensors and Actuators A: Physical 220, 134-143.

Lunov, O., Zablotskii, V., Syrovets, T.,Röcker, C., Tron, K., Nienhaus, U. and Simmet, T. (2011). Modeling receptor-mediated endocytosis of polymer-functionalized iron oxide nanoparticles by human macrophages, Biomaterials 32(2), 547-555. 
Menad, N., Kanari, N. and Save, M. (2014). Recovery of high grade iron compounds from LD slag by enhanced magnetic separation techniques, International Journal of Mineral Processing $126,1-9$

Murariu, V. (2013). Simulating a Low Intensity Magnetic Separator Model (LIMS) using DEM, CFD and FEM Magnetic Design Software, Computational Modelling, June 18-19, Falmouth, UK.

Nopola, T. (2004). Simulation of Particle Motion in Concentrated Colloidal Suspensions, Lic. Thesis, Department of Mathematics, University of Turku, Turku, Finland.

Rosengart, A., Kaminski, M., Chen, H., Caviness, P., Ebner, A. and Ritter, J. (2005). Magentizable implants and functionalized magnetic carriers: A novel approach for noninvasive yet targeted drug delivery, Journal of Magnetism and Magnetic Materials 293(1): 633-638.

Sand, A. (2010). Microscopic Simulation of Pigment Coating Consolidation, Doctoral thesis, Åbo Akademi University, Uniprint, Turku, Finland.

Sand, A., Nopola, T. Hjelt, T. and Toivakka, M. (2009). A particle motion model for the study of consolidation phenomena, Computers and Chemical Engineering 33, 1227-1239.

Sand, A., Toivakka, M. and Rosenkranz, J. (2011). A Modified Stokesian Dynamics Method for Mineral Suspensions, Particles 2011: II International Conference on Particle Methods Fundamentals and Applications, October 26-28, Barcelona, Spain, Conference CD.

Satoh, A. (2002). Development of Effective Stokesian Dynamics Method for Ferromagnetic Colloidal Dispersions (Cluster-based Stokesian Dynamics Method), J. Colloid Interf. Sci. 255, 98-106. 
Satoh, A. (2008). Three-dimensional Monte Carlo simulations of internal aggregate structures in a colloidal dispersion composed of rod-like particles with magnetic moment normal to the particle axis, Journal of Colloid and Interface Science 318, 68-81.

Satoh, A., Chantrell, R., Coverdale, G. and Kamiyama S.-I. (1998). Stokesian Dynamics Simulations of Ferromagnetic Colloidal Dispersions in a Simple Shear Flow, J. Colloid Interf. Sci. 203, 233-248.

Satoh, A., Chantrell, R., Kamiyama, S.-I. and Coverdale, G. (1996). Two-Dimensional Monte Carlo Simulations to Capture Thick Chainlike Clusters of Ferromagnetic Particles in Colloidal Dispersions, J. Colloid Interf. Sci. 178, 620-627.

Sierou, A. and Brady, J. (2001). Accelerated Stokesian Dynamics Simulations, Journal of Fluid Mechanics 448, 115.

Stener, J., Carlson, J., Pålsson, B. and Sand, A. (2014). Evaluation of the applicability of ultrasonic velocity profiling in conditions related to wet low intensity magnetic separation, Minerals Engineering 62, 2-8.

Svoboda, J. and Fujita, T. (2003). Recent developments in magnetic methods of material separation, Miner. Eng. 16(9), 785-792.

Toivakka, M. (1997). Simulation of Particle Motion in Pigment Coating Colors, Doctoral thesis, Laboratory of Paper Chemistry, Åbo Akademi University, Turku, Finland.

Toivakka, M., Salminen, P., Chonde, Y. and Bousfield, D. (1997). Consolidation of Particulate Suspensions - Model Study with Plastic Pigments, TAPPI Advanced Coating Fundamentals Symposium, May 9-10, Philadelphia, PA, TAPPI Press, Atlanta, GA, 89. 
Yung, K., Landecker, P. and Villani, D. (1998). An Analytical Solution for the Force Between Two Magnetic Dipoles, Magnetic and Electrical Separation 9, 39-52.

Wills, B. and Napier-Munn, T. (2006). Mineral Processing Technology - An Introduction to the Practical Aspects of Ore Treatment and Mineral Recovery, $7^{\text {th }}$ Ed., Butterworth-Heinemann, ISBN 978-0-7506-4450-1. 